\title{
Preface to SPECIAL ISSUE: Advances in Metallic Biomaterials
}

Metallic biomaterials, especially advances in various biodegradable metallic materials and porous or open-cellular metallic materials having important applications as orthopaedic and other biomedical implants or appliances, have become the focus of many research groups worldwide. This Special Issue themed on "Advances in Metallic Biomaterials" presents not only a representative segment of eminent researchers in areas related to metallic biomaterials, but also leading edge innovations. Papers included in this Special Issue represent novel and advanced biomaterials research as well as reviews and overviews of critical developments and emerging technologies involving biomedical materials. Topics covered include basic research and applications involving biomaterials processing and fabrication, biomedical and biomechanical properties of novel implant materials, in-vitro and in-vivo studies or performance issues, clinical trials, and strategies for rendering porous structures functional, living scaffolds.

Six of the sixteen papers composing this Special Issue highlight Ti-alloy implants and related appliances utilizing $3 \mathrm{D}$ printing/additive manufacturing, including functional, porous implants. Another six papers describe innovations in biodegradable $\mathrm{Mg}$ or Mg-alloy stents and bone-replacement metallic implants, with emphasis on the corrosion, mechanical and biocompatible performances. In the rest of the papers, strategies for inducing osseointegration and vascularization in porous, opencellular metallic implants are discussed along with bioactive and related surface treatments and modifications, and Ti scaffold functionalization. Biodegradable bone screws of $\mathrm{Fe}$ and 316L stainless steel fabricated by friction-stir processing are also described.

The Editors hope that this Special Issue will inspire new metallic biomaterials innovations and future developments by serving as a general overview of current advances and state-of-the-art in metallic biomaterials research worldwide.

\section{Guest editors}

Professor Lawrence E. Murr

Department of Metallurgical, Materials and Biomedical Engineering, University of Texas at El Paso, USA Email: lemurr@utep.edu

Professor Cuie Wen School of Engineering, RMIT University, Australia Email: cuie.wen@rmit.edu.au

Professor Yufeng Zheng

Department of Materials Science and Engineering, College of Engineering, Peking University, China Email: yfzheng@pku.edu.cn 